\title{
Novel Low-Cost MIMO-RoF-PON Architecture for Next Generation Integrated Wired and Wireless Access Networks
}

\author{
T.H.DAHAWI, ${ }^{1}$ Z.YUSOFF, $^{1 *}$ M.S.SALLEH, ${ }^{2}$ J.M.SENIOR. ${ }^{3}$
}

1 Multimedia University, Persiaran Multimedia, 63100 Cyberjaya, Malaysia

2 TM R\&D, Lingkaran Teknokrat Timur, Cyberjaya, Malaysia.

3 Optical Networks Group, University of Hertfordshire, Hatfield,AL10 9AB, United Kingdom,

*Corresponding author: zulfadzli.yusoff@mmu.edu.my

Received XX Month XXXX; revised XX Month, XXXX; accepted XX Month XXXX; posted XX Month XXXX (Doc. ID XXXXX); published XX Month XXXX

\begin{abstract}
Next generation integrated fiber-wireless access networks will require low-cost and high capacity deployment to meet the customer demand. A new configuration of radio over fiber-passive optical network (RoF-PON) architecture, including two $60 \mathrm{GHz}$ multiple-input multiple-output (MIMO) based on a 5G universal filtered multicarrier (UFMC) waveform and wired signal utilizing orthogonal frequency division multiplexing (OFDM), is described. At the optical line terminal MIMO signals are integrated as upper and lower sidebands of the wired OFDM signal. This integration approach employing single sideband frequency translation (SSB-FT) reduces the complexity of the transceiver design and provides high spectral efficiency because the two MIMO-RoF and wired signals transmit at the same frequency. Improved techniques are also employed to upconvert and downconvert the $60 \mathrm{GHz}$ millimeter wave (MMW) being remote optical heterodyning and self-heterodyne, respectively. The MIMO-RoF signals are therefore transmitted at low frequency over the standard single mode fiber to avoid the impairments induced at higher frequencies and the remote optical local oscillator is reused to downconvert the two $60 \mathrm{GHz}$ MMWs producing a costeffective system. Simulation results demonstrate very satisfactory network performance when using a downstream link over a $20 \mathrm{~km}$ span standard-PON.
\end{abstract}

Index Terms - 5G, Millimeter wave, RoF, OFDM-PON, SSB-FT and MIMO-UFMC.

http://dx.doi.org/10.1364/JOCN.99.099999

\section{INTRODUCTION}

Recently, the great interest in Internet of Things applications, together with the increased demand for high-speed streaming necessitate a complete shift in shaping the future 5G mobile network [1]. New waveforms have been discussed for $5 \mathrm{G}$ implementation, which provide improved time-frequency localization to support the connection flexibility to numerous users to replace orthogonal frequency division multiplexing (OFDM) [2]. In addition, the utilization of the millimeter wave (MMW) bands is considered to have promising potential for $5 \mathrm{G}$ technology that requires ultra-dense deployment of small cells in synchronization with cloud radio access network (C-RAN) [2]. Hence the requirement for providing high capacity mobile fronthaul and backhaul links is clearly important.

A Radio over fiber (RoF) system including a fiber distribution network could be implemented as the optical transport portion for $5 \mathrm{G}$ network which provides high capacity, low latency and allows efficient fronthauling and backhauling for wireless signals [3]. Moreover, since $5 \mathrm{G}$ systems transmit in the higher frequency MMW band, where the generation of MMW frequencies creates less cost, as it necessitates simple photonic techniques compared to costly and highly complex electronic components if a none-RoF system is implemented [4]. To reduce the capital expenditure (CAPEX) of RoF installation, it is suggested that the RoF system should be converged with currently deployed optical access networks using a passive optical network (PON), which provides a fiber infrastructure that can be utilized for backhauling $5 \mathrm{G}$ to end users at affordable cost [5].

In order to converge a RoF based-5G system with a PON, the 5G candidate waveform should comply with $5 \mathrm{G}$ features. Universal filtered multicarrier (UFMC) is one of the highly spectral efficient waveforms designed for the $5 \mathrm{G}$ wireless network [6]. It is a modified form of OFDM, where a filtering technique is utilized on a group of subcarriers to adjust the spectral properties of its signal, resulting in a lower out-of- band transmission compared to OFDM [7]. UFMC has been extensively analyzed and evaluated in wireless domain [8-13] but little research has targeted UFMC performance in optical domain. Nevertheless UFMC was evaluated in an analogue MMW RoF system and intermediate frequency (IF) over fiber. However in [14], the UFMC-RoF in both cases were not integrated with a standard PON leading to high cost systems. In [15], although UFMC-RoF was converged with a standard PON based on a single carrier 4-PAM signal, it is of more interest to investigate UFMC performance in terms of the convergence with the wired PON signals based on multicarrier waveforms such as that employed in an OFDM-PON. This situation is created because the intermixing between 
the two waveforms (UFMC and OFDM) will affect the performance of the integrated network $[14,16]$. In addition, it is important to implement the multiple-input multiple-output (MIMO) technique in the millimeter-wave band for next generation-PON as it will be used for fronthauling the future 5G systems [17], which provides improved system reliability $[18,19]$. The integration of MIMO-RoF and a PON can therefore be achieved using an architecture that creates a resourcesharing platform, producing a cost-effective integrated network.

Several integrated network architectures enabling wired and wireless services to operate together have been reported [20-24]. When in $[20,21]$, the wired (wavelength division multiplexed (WDM)-PON) and wireless (MMW RoF) services are modulated on different wavelength signals it results in an expensive system if the deployment of a MIMO is also required. In [24] the wired OFDM and $60 \mathrm{GHz}$ MMW wireless signals are integrated and modulated with the same wavelength signal which enables improved resource-sharing optimization in comparison with the schemes proposed in [20, 21]. However, when the two MIMO approach is considered there is a need for another optical source and modulator. This in turn creates a complex system. A RoF-PON integrated architecture including MIMO signals based on polarization multiplexing is described in [22, 23]. This approach enables both 16 QAM MIMO-ROF and baseband wired-PON signals to be transported over a single wavelength signal. Nevertheless, each MIMO and wired signal require an independent optical modulator and it also necessitates a polarization controller for each MIMO creating an expensive system.

It is more attractive to deploy a 5G-based RoF-PON that supports wired and MMW MIMO wireless signals on the same infrastructure such as in the implementation of an OFDM-PON [5] which provides an equipment-sharing platform resulting in a low cost convergent network. Thus the objective of this paper is to design a new low-cost architecture for the integration of RoF based on MMW MIMO-UFMC and OFDM-PON. In the proposed approach a single sideband frequency translation (SSB-FT) technique [25] is utilized to enable wired and two MIMO wireless signals to be upconverted at same carrier frequency via operating only one local oscillator (LO) at the transceiver designed to shift the two MIMO signals to left and right of the wired signal. This leads to the use of only one laser source and also one optical modulator to modulate both the wired and the two MIMO signals at a single wavelength without utilizing a WDM multiplexer and demultiplexer. The proposed architecture operates with the simple intensity modulation and direct detection (IM-DD) scheme where remote and self-heterodyne techniques [26] are implemented to generate the matched $60 \mathrm{GHz}$ local oscillator signal reducing the overall cost and design complexity of $5 \mathrm{G}$ remote radio heads (RRHs) and user equipment (UE). In addition, the co-existence of both 5G UFMC and OFDM waveforms are explored.

The paper is organized as follows. Section 2 elaborates the principle of the proposed 5G-based RoF-PON architecture then in section 3, the description and modelling of the network configuration are presented. The results and discussion associated with the modelling are then covered in section 4 . Finally, section 5 provides conclusions, while a theoretical analysis providing confidence in relation to the simulation performance is provided in an Appendix.

\section{UFMC and System Operating Principles}

The universal filtered multicarrier waveform has been chosen due to its superiority in wireless convergence with the PON compared to the other $5 \mathrm{G}$ candidate waveforms such as the generalized frequency division multiplexing (GFDM) and filtered-bank multicarrier (FBMC) [27]. In addition, UFMC is more tolerant to interband interference, so that multiple bands and services can be supported in RoF system [14]. Moreover, UFMC provides more efficient MIMO integration which leads to reduction in the complexity of the beamforming system [28]. Subsystems such as single sideband frequency translation (SSB-FT) and remote optical heterodyne MMW upconversion techniques have been utilized to provide an efficient and cost-effective converged network $[25,26]$. The SSB-FT approach enables simultaneous transmission of MIMO streams and wired OFDM-PON signals over the PON infrastructure without the need for a WDM system. However, using the remote optical heterodyne MMW upconversion technique, MIMO-RoF signals are transmitted at low frequency over the standard single mode fiber (SSMF) to avoid the impairments induced at high frequencies. UFMC theory and the subsystem operational principles are dealt with in the following subsections.

\section{A. UFMC Theory}

UFMC is considered to be new candidate $5 \mathrm{G}$ waveform $[29,30]$ due to its significant features such as high spectral efficiency, low out of band emission and low peak-to-average power ratio (PAPR) compared to conventional OFDM [28]. The UFMC digital signal processing structure is similar to OFDM as it is digitally implemented using the inverse fast Fourier transform (IFFT) [19]. The signal bandwidth of UFMC, however, is split into different subbands which are equivalent to resource blocks (RBs) in long term evolution (LTE) systems containing the allocation of the LTE frame that is divided based on the time and subcarrier frequency axis [31]. The subbands are linearly filtered to tackle the outof-band emission which causes an in-band leakage problem in the OFDM signal that causes significant deterioration in the performance [32].

The basic transceiver model to produce UFMC is illustrated in Fig. 1 [27]. The data QAM symbols are mapped to allocate sub-bands for a given user. Each subband is transformed to the time domain using an $\mathrm{N}$ point IFFT module and then processed by a Dolph-Chebyshev band pass filter [33]. The superposition of the subband filtered signals is defined as the UFMC transmitted signal that is denoted by vector $X$ and expressed by the following [34]:

$$
X=\sum_{b=1}^{B} S_{b} V_{b} F_{b}
$$

where $S_{b}$ is the vector of complex QAM symbol for each subband, where $b=(1,2,3, \ldots, B) . V_{b}$ is a vector of dimension $N \times k_{b}$ that includes the respective columns of the IFFT matrix for each subband position within the overall frequency range; $N$ equals to the total number of subcarriers and $\mathrm{k}_{\mathrm{b}}$ is the allocated subcarriers for each subband. $F_{b}$ is a Toeplitz matrix with dimension $\left(N+N_{\text {filter }}-1\right)$ which contains the filter impulse response that enables the convolution.

The UFMC transmitted signal is passed through the channel and the demodulation is carried out using two processes. In the first process, the $\left(N+N_{\text {filter }}-1\right)$ received signal samples are zero padded to the length $2 N$, then a $2 N$ point Fast Fourier Transform (FFT) operation is performed. For the second process, a frequency domain equalizer (FDE) is applied to equalize the distorted signal. Finally, the received bits can be estimated after the symbol de-mapping process. 


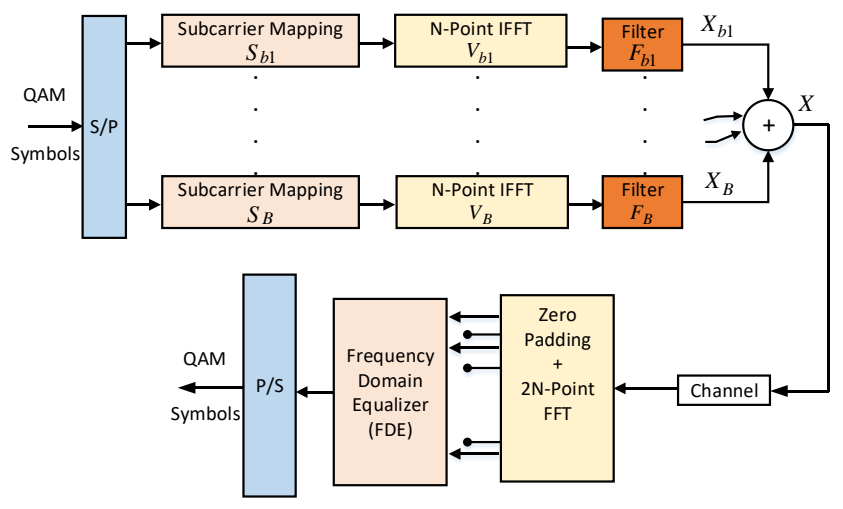

Fig. 1. UFMC transceiver.

\section{B. Single Sideband Frequency Translation Technique}

Transporting MIMO signals over a RoF-PON integrated network is challenging due to spectrum overlapping [35]. A straightforward solution is to assign different MIMO channels to specific fibers. However, this is not cost effective. Therefore a multiplexing technique is required to deliver them simultaneously to the RRH using a single SSMF. Although wavelength division multiplexing, subcarrier multiplexing (SCM) and polarization division multiplexing (PDM) are techniques that have been used for transmitting MIMO signals over a single SSMF [23, $36,37]$, these techniques are expensive and complex . Using a WDM system then an optical source and photodetector are required for each MIMO channel [37]. In an SCM system $n$ - 1 local oscillators (LOs) are needed for $n$ MIMO channels [36]. In addition, when using PDM technique polarization controllers and independent optical modulators are required for the MIMO signals and wired PON signals [23].
A single sideband frequency translation (SSB-FT) technique was reported in [25] to tackle the above limitation of SCM. It was proposed for transmitting three MIMO signals over a single optical fiber utilizing only one LO. In this case one MIMO-channel was considered as the central band and the other two MIMO channels were located as left and right sidebands at the same carrier frequency. Hence the technique was referred to as single sideband frequency translation. This approach was utilized in the new RoF-PON integrated architecture to enable wired signal and two MIMO-RoF channels to be transmitted over a standard OFDM-PON to both reduce the cost and to improve the spectral efficiency.

Fig. 2 shows the implementation of the adapted SSB-FT technique in the new network architecture which was significantly adapted from the architectures reported in $[25,38]$. At the optical line terminal (OLT), two MIMO channels are multiplexed together. A LO signal frequency $f_{L O}$ drives the two mixers which results in shifting each MIMO channel into upper and lower sideband of the original RF frequency $f_{R F}$. Then two bandpass filters (BPFs) are used to remove the unwanted band for each MIMO channel. For MIM01, BPF with center frequency equivalent to $f_{R F}+f_{L O}$ is used to select the translated upper sideband frequency, whereas another BPF with center frequency equivalent to $f_{R F}-f_{L O}$ is utilized to select the translated lower sideband frequency for MIMO2. Then the two MIMO channels associated with the wired-PON signal which is upconverted to $\mathrm{RF}$ frequency equivalent to $f_{R F}$. The aggregated signal, namely; the wired-PON and MIMO signals will be then optically modulated by the electrical-to-optical converter (E/O) to be transported through the PON infrastructure to different users.
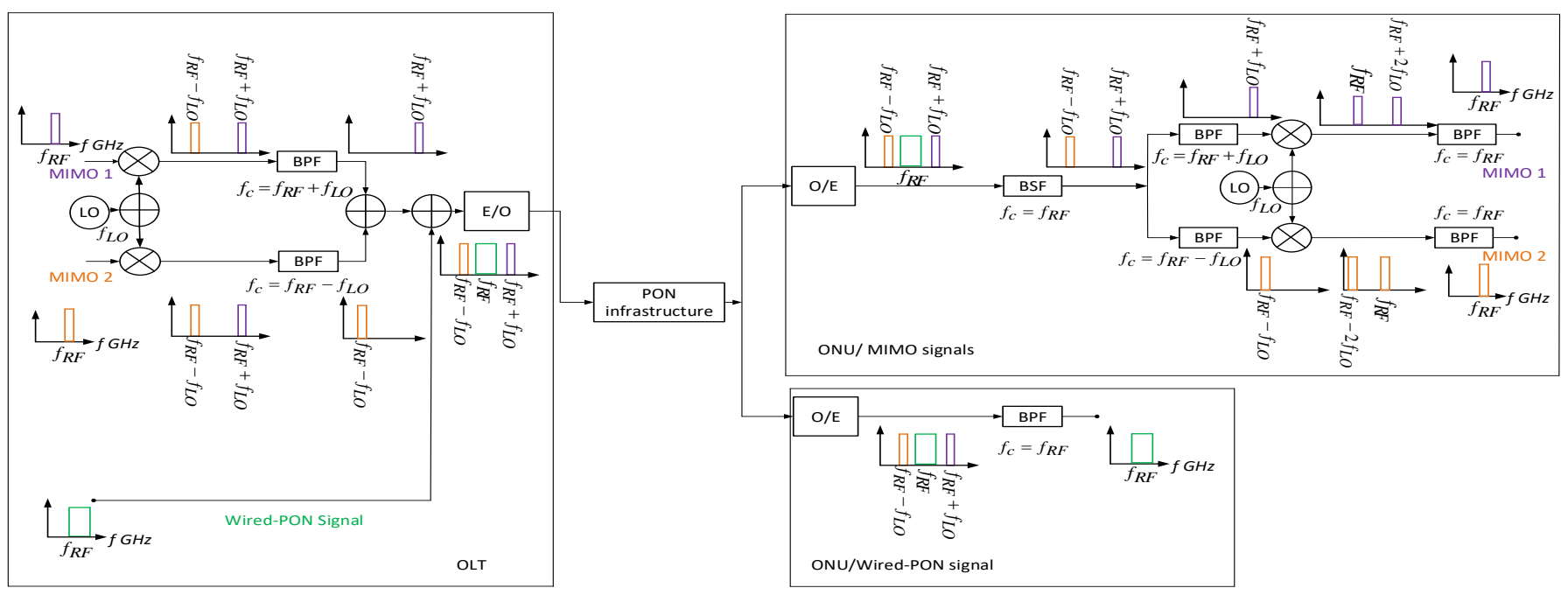

Fig. 2. Implementation of SSB-FT technique over the new architecture.

The double sideband frequency translation (DSB-FT) approach established in reference [38] was applied to the new architecture shown in Fig. 2. In the new architecture the LO signal drives the two mixers without requiring a $90^{\circ}$ hybrid coupler as in [38]. Furthermore in Fig. 2 the wired-PON signal is associated with the two MIMO systems. Hence similar to the derivation in [38], after mixing the LO signal with the two MIMO signals they can be expressed as:

$$
M I X_{\text {out } M 1}=m_{1} \sin \left(2 \pi f_{R F} t+\theta_{1}\right) \sin \left(2 \pi f_{L O} t\right)
$$

$$
M I X_{\text {outM } 2}=m_{2} \sin \left(2 \pi f_{R F} t+\theta_{2}\right) \sin \left(2 \pi f_{L O} t\right)
$$

where $M I X_{\text {out } M 1}$ and $M I X_{\text {out } M 2}$ are the outputs of the two mixers dedicated to MIM01 and MIM02, respectively. The messages in MIM01 and MIMO2 are denoted as $m_{1}, m_{2} . \theta_{1}, \theta_{2}$ are the initial phases for MIM01 and MIM02, respectively. Frequencies for radio signals and local oscillator are represented by $f_{R F}$ and $f_{L O}$ and therefore equations (2) and (3) can each be expanded using trigonometric Identities as: 


$$
\begin{aligned}
& \left.M_{\text {II } M 1}=\frac{1}{2}\left\{\begin{array}{l}
m_{1} \cos \left[\left(2 \pi f_{R F}-2 \pi f_{L O}\right) t+\theta_{1}\right] \\
-m_{1} \cos \left[\left(2 \pi f_{R F}+2 \pi f_{L O}\right) t+\theta_{1}\right.
\end{array}\right]\right\} \\
& \left.M I X_{\text {out } M 2}=\frac{1}{2}\left\{\begin{array}{l}
m_{2} \cos \left[\left(2 \pi f_{R F}-2 \pi f_{L O}\right) t+\theta_{2}\right] \\
-m_{2} \cos \left[\left(2 \pi f_{R F}+2 \pi f_{L O}\right) t+\theta_{2}\right.
\end{array}\right]\right\}
\end{aligned}
$$

The multiplexed MIMO channels $M_{m u x}$ obtained after applying the BPFs are expressed by the following:

$$
M_{\text {mux }}=\frac{1}{2}\left\{\begin{array}{l}
m_{2} \cos \left[\left(2 \pi f_{R F}-2 \pi f_{L O}\right) t+\theta_{2}\right] \\
-m_{1} \cos \left[\left(2 \pi f_{R F}+2 \pi f_{L O}\right) t+\theta_{1}\right]
\end{array}\right\}
$$

The combined signal $M_{T x}$ as depicted in Fig. 2 contains the MIMO and wired-PON signals and can be written as:

$$
M_{T x}=\frac{1}{2}\left\{\begin{array}{l}
m_{2} \cos \left[\left(2 \pi f_{R F}-2 \pi f_{L O}\right) t+\theta_{2}\right] \\
+m_{3} \sin \left[\left(2 \pi f_{R F}\right) t+\theta_{3}\right] \\
-m_{1} \cos \left[\left(2 \pi f_{R F}+2 \pi f_{L O}\right) t+\theta_{1}\right]
\end{array}\right\}
$$

where $m_{3}$ and $\theta_{3}$ represent the message and the initial phase of wiredPON signal respectively.

At the optical network units (ONUs) as shown in Fig. 2 the optical transmitted signal is distributed to different users and an optical-toelectrical converter $(\mathrm{O} / \mathrm{E})$ is required for each $\mathrm{ONU}$ to convert to an electrical signal. A BPF is required to select the wired-PON signal that is dedicated to the wired service. At the ONU dedicated for the MIMO channels a band stop filter (BSF) is utilized to remove the unwanted wired-PON signal and then the reverse of the SSB-FT process is applied to detect the MIMO signals at their original frequencies.

\section{Remote Optical Heterodyne for 5G MMW Upconversion}

There are two methods to upconvert the MMW in RoF-PON those being direct and remote upconversion [39]. In direct upconversion, the MMW signal is generated at the OLT and directly transmitted through the fiber link [40]. However, the transmission of a high frequency signal over the length of the PON branches incurs a large power penalty due to chromatic dispersion effects. Furthermore transmitting MMW through the PON will require high-speed optical components [41]. To solve the direct upconversion problem, a low frequency signal transport scheme over the PON can be used to reduce a large power penalty due to chromatic dispersion [42]. This can be done by implementing a remote MMW upconversion using a heterodyne technique [26]. Hence the optical heterodyne technique is one of the methods that is used for the generation of the MMW signals through beating of the two optical carriers with a wavelength spacing corresponding to the desired MMW frequency [43].

In the new architecture the remote optical heterodyne technique is employed as illustrated in Fig. 3 where a remote optical local oscillator is coupled with the modulated combined signal that was identified in the section 2 B. The combined spectrum is shown as in inset (a) of Fig. 3. The wavelength of the optical LO $\lambda_{L O}$ is determined based on which MMW frequency is used. For example, in this case a $60 \mathrm{GHz}$ MMW frequency was utilized so that the distance between the reference optical carrier $\lambda_{T X}$ and the optical LO carrier must be equal to $60 \mathrm{GHz}$. This is illustrated through the output spectrum after the coupler in inset (b) of Fig. 3. The output spectrum after the coupler includes the modulated data at intermediate frequency and unmodulated optical LO signal that are transmitted through SSMF and then the optical transmitted signal is distributed to different users. The photodetector (PD) at the RRH produces the modulated data at the MMW frequency that is shown in inset (c) of Fig. 3. Before transmitting the upconverted MMW signals through the antenna, BSF is used to suppress the wiredPON signal as displayed in inset (d) of Fig. 3. The MIMO signals at $60 \mathrm{GHz}$ MMW bands are then amplified using the electrical amplifier (EA) and transmitted to the user.

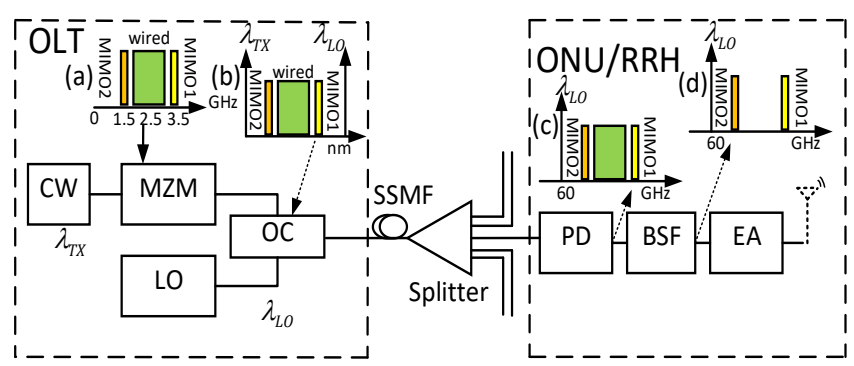

Fig. 3. Schematic diagram for MMW upconversion.

\section{Network Description and Modelling}

Fig. 4 shows the simulation configuration for the downstream transmission of the new $5 \mathrm{G}$ RoF-PON architecture including two $60 \mathrm{GHz}$ MIMO-UFMC and wired-OFDM based on a single sideband frequency translated technique using the industry standard VPI and MATLAB software [44].

\section{A. Signal Generation}

The transmitted UFMC waveform for each MIMO signal at the intermediate frequency (IF) band is generated in two steps as shown in Fig. 4. The first step is the design of the digital signal processing (DSP) that manipulates the transmitted signal in digital domain and it was performed off-line using MATLAB software. Furthermore, the generic diagram of this DSP procedure can be seen in Fig. 1. A next step is the digital to analog conversion (DAC) which produces a complex-valued baseband UFMC signal with I and Q branches that are modulated and upconverted by a pair of IF signals through I/Q mixer to output a realvalued IF-UFMC signal. The IF frequency in the simulation is $2.5 \mathrm{GHz}$. The UFMC DSP produced from MATLAB was loaded into VPI creating the DSP to DAC and the upconversion process to generate $2.5 \mathrm{GHz}$ UFMC signal. The specification of the UFMC waveform is shown in Table 1 where there is a total of 12 subbands in which 26 subcarriers are modulated on 16 quadrature amplitude modulation (16 QAM) for each subband. In addition, the data rate of the UFMC signal is $1 \mathrm{Gbps}$. The wired OFDM signal was generated using the OFDM models within VPI and it was modulated on 16 QAM with a $1.25 \%$ cyclic prefix (CP) and the signal data rate and bandwidth were $2.5 \mathrm{Gbps}$ and $0.625 \mathrm{GHz}$, respectively. Following, the wired OFDM signal was upconverted with the same IF carrier as UFMC at $2.5 \mathrm{GHz}$ and it was then added to the two MIMO-UFMC signals using the single sideband frequency translated process. 


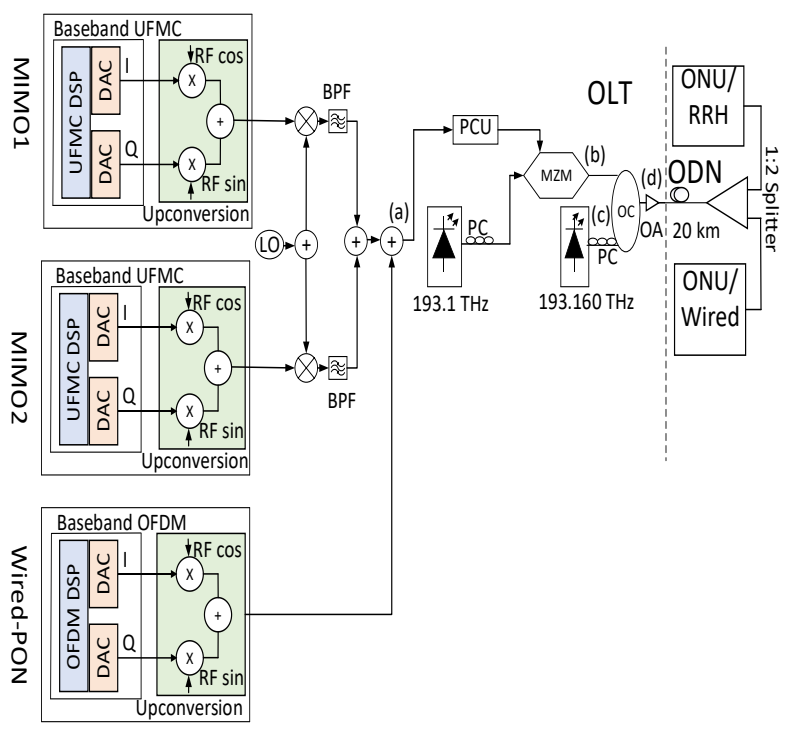

Fig. 4. Simulation configuration for the new network architecture.

TABLE 1

UFMC Properties

\begin{tabular}{|l|l|}
\hline IFFT size & 512 \\
\hline Subcarriers & 26 \\
\hline Sub-bands & 12 \\
\hline Filter type & Dolph-Chebychev \\
\hline Filter length & 46 \\
\hline Modulation & $16-\mathrm{QAM}$ \\
\hline Data Rate & $1 \mathrm{Gbps}$ \\
\hline Bandwidth & $0.25 \mathrm{GHz}$ \\
\hline
\end{tabular}

\section{B. 5G-based PON configuration}

At the OLT shown in Fig. 4, the MIMO-UFMC signals and wired OFDM signal are generated as outlined in Section 3A where all signals are upconverted to the same frequency of $2.5 \mathrm{GHz}$. The simulation design parameters for this process are provided in Table 2.

TABLE 2

Simulation Design Parameters

\begin{tabular}{|l|l|}
\hline Parameter & Value \\
\hline Data Rate for OFDM-PON & $2.5 \mathrm{Gbps}$ \\
\hline Data Rate for each UFMC signal & $1 \mathrm{Gbps}$ \\
\hline Carrier frequency & $2.5 \mathrm{GHz}$ \\
\hline Modulation & $16 \mathrm{QAM}$ \\
\hline LO frequency & $1 \mathrm{GHz}$ \\
\hline Bandwidths for all BPFs & $1 \mathrm{GHz}$ \\
\hline Bandwidth of BSF & $1.5 \mathrm{GHz}$ \\
\hline Laser power & $15 \mathrm{dBm}$ \\
\hline Laser linewidth & $100 \mathrm{KHz}$ \\
\hline MZM extinction ratio & $15 \mathrm{~dB}$ \\
\hline Fiber length & $20 \mathrm{~km}$ \\
\hline Fiber attenuation & $0.2 \mathrm{~dB} / \mathrm{km}^{2}$ \\
\hline Dispersion Coefficient & $17 \mathrm{e}-6 \mathrm{~s} / \mathrm{m}^{2}$ \\
\hline PD responsively & $1 \mathrm{~A} / \mathrm{W}$ \\
\hline PD type & $\mathrm{APD}$ \\
\hline
\end{tabular}

By utilizing the single sideband frequency translation technique (SSB-FT), MIMO 1 is mixed by a $1 \mathrm{GHz}$ local oscillator which produces an upper sideband at $3.5 \mathrm{GHz}$ and a lower sideband at $1.5 \mathrm{GHz}$ and other higher order sidebands at different frequencies. A bandpass filter with center frequency $f_{C}=3.5 \mathrm{GHz}$ was used to select the upper sideband at 3.5 GHz. MIMO 2 signal was also mixed with $1 \mathrm{GHz}$ LO to produce the same spectrum as MIMO 1 and thus a BPF with $f_{C}=1.5 \mathrm{GHz}$ can be used to select the lower sideband from the MIMO 2 signal. After the mixing process, the original frequency at $2.5 \mathrm{GHz}$ was left unoccupied so that the wired-OFDM signal can be inserted as displayed in Fig. 5 (a).
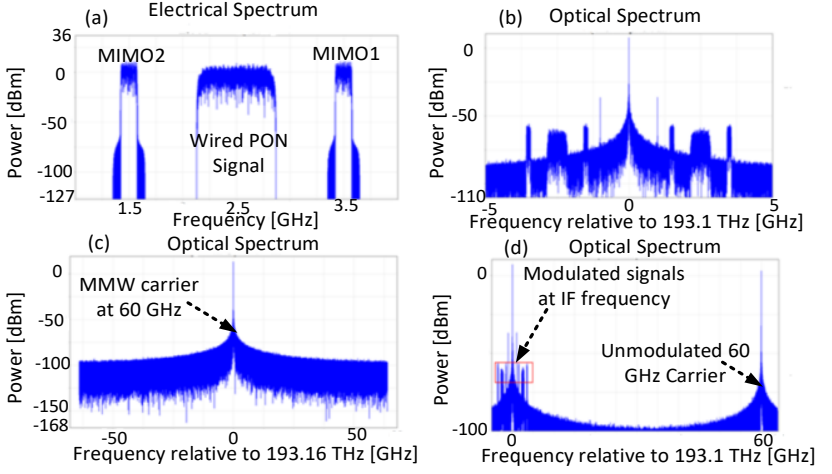

Fig. 5 (a)-(d). The spectra of different optical and electrical signals at assigned points in Fig. 4.

The combined electrical signal was then fed by a power control unit (PCU) to a Mach-Zehnder modulator (MZM) RF input biased at its quadrature point, which is set to be $5.4 \mathrm{~V}$. A PCU was utilized to adjust the input RF power inside MZM to avoid non-linearity issues that produce intermodulation distortion causing the degradation of the system performance. A continuous wave laser signal at $193.1 \mathrm{THz}$ frequency, $100 \mathrm{KHz}$ linewidth and $15 \mathrm{dBm}$ output power was subject to 3-stages polarization control (PC) and then fed into the second arm of the MZM to modulate the combined signals. Fig. 5 (b) shows the optical spectrum after MZM, which comprises optical double-sidebands (ODSB) modulation with $f_{C}=193.1 \mathrm{THz}$. since the standard PON span is $20 \mathrm{~km}$ and also the signal is transmitted with low frequency through SSMF then the chromatic dispersion does not have a significant effect on the performance [45] and hence the reason why ODSB was chosen to avoid the need for a narrow optical filter after MZM which will increase the cost of the network. It is important to point out that the peaks which can be seen at right and left side of the optical carrier shown in Fig. 5 (b) are caused by the local oscillator pulse that was used in the SSB-FT process shown in Fig. 2. Although they could be removed using the bandpass filters, the filters that were utilized had a bandwidth greater than the bandwidth of the MIMO-UFMC signals to avoid the interference caused by the leaking issues as a consequence of the SSB-FT process [25]. Their presence, however, is not significant as they do not affect the modulated signal and also carry no data.

The output of the MZM was then combined with local oscillator laser at $193.160 \mathrm{THz}$ as shown in Fig. 5 (c) using a 3-dB optical coupler. Furthermore, the difference in the two laser frequencies (the reference frequency and the local oscillator laser frequency) is equivalent to the $60 \mathrm{GHz}$ MMW that is displayed in Fig. 5 (d). Then the combined modulated RF signals and unmodulated $60 \mathrm{GHz}$ MMW carrier were amplified through a controlled power optical amplifier (OA) to ensure the power inserted to the SSMF was $5 \mathrm{dBm}$ in order to reduce the fiber nonlinearities that arise at higher input power. 
At the optical distribution network (ODN) the signal is transmitted over SSMF with a wavelength of $1552.524 \mathrm{~nm}$, being equivalent to a frequency of $193.1 \mathrm{THz}$. The power attenuation and chromatic dispersion of the SSMF were $0.2 \mathrm{~dB} / \mathrm{km}$ and $17 \mathrm{ps} / \mathrm{nm} . \mathrm{km}$, respectively. A power splitter with 1:2 spilt ratio was utilized to transmit the optical signal to two ONUs that were connected to the wired-OFDM and remote radio head for the two $60 \mathrm{GHz}$ MIMO-UFMC wireless signals.

The ONU for the wired-OFDM signal is shown in Fig. 6 where the received optical signal from the ODN is injected into a high-speed photodetector for optoelectrical downconversion. The two coupled optical carriers which are shown in Fig. 5 (d) include the reference optical carrier with modulated signals at $193.1 \mathrm{THz}$, and the remote optical carrier at a wavelength equivalent to $193.160 \mathrm{THz}$. When these two optical carriers are applied to the photodetector, a signal that is an exact replica of the original modulated signal but moved to a higher frequency band equivalent to the difference between the two carriers is generated. This phenomenon occurred due to carrier beating between the two optical carriers and is referred to as the optical heterodyne technique [46] which was discussed in section 2C.

In the new architecture, the carrier beating at the PD generates a photocurrent that includes the wired-OFDM signal at $2.5 \mathrm{GHz}$ IF frequency and at $60 \mathrm{GHz}$ MMW band as displayed in Fig. 7 (a). A bandpass filter with $f_{C}=2.5 \mathrm{GHz}$ was used to select the desired wiredOFDM signal shown in Fig. 7 (b) which is further downcoverted to its baseband form and then processed through the digital signal processing, bit error rate (BER) and error vector magnitude (EVM) analyzer.

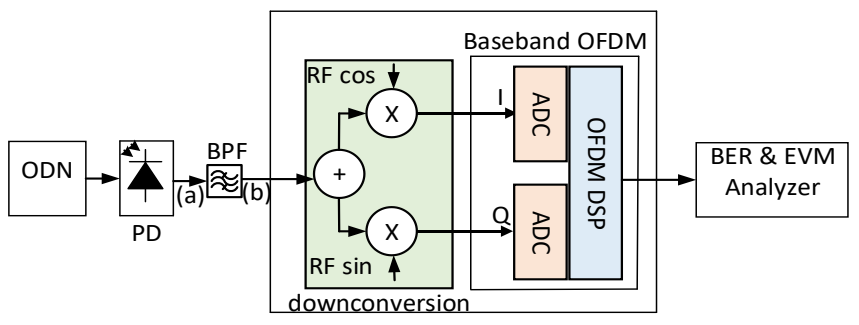

Fig. 6. Simulation configuration of the ONU for the wired-OFDM signal.
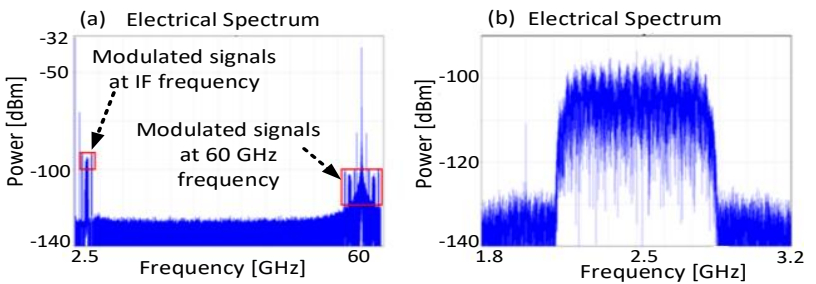

Fig. 7 (a), (b). The spectra of electrical signals at assigned points in Fig. 6.

Fig. 8 displays the configuration for the $\mathrm{ONU}$ dedicated to produce the two $60 \mathrm{GHz}$ MIMO-UFMC signals, where the split optical signal was received from the ODN and thus a high-speed photodetector was used for optoelectrical downconversion. The output electrical spectrum is the same as that shown in Fig. 7 (a) which is caused by the carrier beating at the PD while the photocurrent includes the modulated signals at both $2.5 \mathrm{GHz}$ IF frequency as well as at the $60 \mathrm{GHz}$ MMW band. A BPF with $f_{C}=60 \mathrm{GHz}$ and bandwidth of $10 \mathrm{GHz}$ is used for passing the signals in the MMW band, which is illustrated in Fig. 9 (a). Then the BSF was used to suppress the digitally generated wired-OFDM band before the two $60 \mathrm{GHz}$ MIMO signals were amplified and transmitted through antenna as is displayed in Fig. 9 (b). The electrical amplifier (EA) provides $20 \mathrm{~dB}$ gain. At the user end (UE) the downconversion of the 60 GHz MMW was undertaken using the receiver carrier-self heterodyne technique.

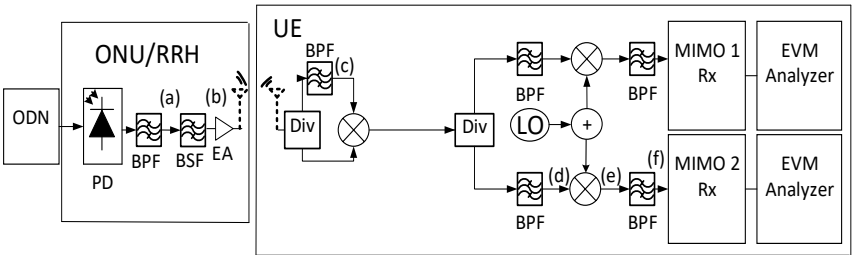

Fig. 8. Simulation configuration of the ONU for the two 60 MIMOUFMC signals.
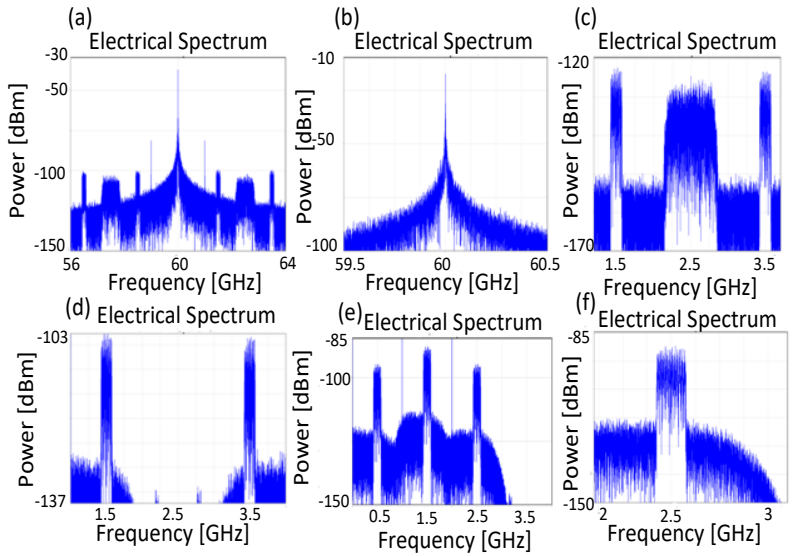

Fig. 9 (a) - (f). The spectra of different electrical signals at assigned points in Fig. 8.

A power divider (Div) splits the received MMW signal so that the 60 GHz MMW carrier is extracted from the received signal at the MMW band by using a narrow BPF with $f_{C}=60 \mathrm{GHz}$ and bandwidth of $2 \mathrm{GHz}$ as shown in Fig. 9 (c). The extracted carrier was then used as the LO for downconverting the $60 \mathrm{GHz}$ MIMO signals to their IF frequencies that are output from the mixer. Therefore the use of a high frequency LO is avoided leading to a reduction in the network cost. After the MMW downconversion, the reverse process using the SSB-FT technique was implemented to convert back the MIMO signals to their original frequencies as displayed in Fig. 9 (d), (e) and (f) that represent the electrical spectra after the SSB-FT process for MIMO 2. Then each MIMO signal was demodulated and the EVM analyzer measured the quality of each MIMO signal. Finally, to verify the performance of the proposed RoF link then the two MIMO-UFMC were directly demodulated without wireless transmission as the implementation of a wireless channel model was not in the scope of this paper.

\section{Network Simulation Performance}

In order to determine the performance of the new networkarchitecture, the downlink fiber transmission performance for both wired-OFDM signal and the two $60 \mathrm{GHz}$ MIMO-UFMC connected in the back-to-back (B-T-B) mode and after $20 \mathrm{~km}$ SSMF transmission were evaluated using EVM and BER measurements. In addition, an investigation on the performance of the two $60 \mathrm{GHz}$ MIMO-UFMC signals before and after convergence with wired-OFDM signal was undertaken in order to measure the power penalty of the RoF system in the converged network. However, before evaluating of the system performance, the RF drive power into MZM was optimized via the power control unit to 
reduce the effect of nonlinearities in the optical modulator that causes the interband intermodulations leading to an interference contribution from both multicarrier waveforms [21]. The analysis of the results is divided into the following three sub-sections.

\section{A. Drive Power Optimization}

The EVM values at different RF drive power for UFMC MIM01, MIMO2 and OFDM-wired signals after $20 \mathrm{~km}$ SSMF transmission are displayed in Fig. 10. It can be noted that the EVM performance increases for higher values of RF drive power around $10 \mathrm{dBm}$ due to the increase in power from the higher order sidebands of the modulated output causing harmonic distortion. As a consequence, interband intermodulations between the OFDM-wired signal and the two MIMO-UFMC RoF signals will occur resulting in the reduction of the signal to noise ratio (SNR) for the network. On the left hand side of Fig. 10 the EVM characteristics are also higher with lower RF drive power due to lower modulation index that degrades the SNR. Therefore, for the new network architecture an optimum power of $-2 \mathrm{dBm}$ was chosen since it has a lower EVM value for all signals which are $1.5 \%, 2.9 \%$ and $3.6 \%$ for wired, MIMO1 and MIMO2 signals, respectively.

At the optimum power of $-2 \mathrm{dBm}$, the EVM performance of wiredOFDM signal is slightly improved over the performance of the MIMOUFMC signals. It was caused by the effect of SSB-FT process on the translated MIMO-UFMC signals compared to untranslated wired-OFDM signal. This improvement was experimentally demonstrated in [25] for the untranslated MIMO3 when it was compared with the translated MIM01 and MIMO2 signals. In addition, it can be seen in Fig. 10 that when the drive power increases the EVM performance of the MIMOUFMC signals increases to a much higher level than wired-OFDM signal. This situation indicates that the intermodulation product or the MZM nonlinearity has a greater effect on the SSB-FT process implemented in MIMO-UFMC signals which leads to the system performance degradation.

The trend of the characteristics in Fig. 10 can also be compared with the results obtained from an experimental study in [47], where interband intermodulation of multiband OFDM signals which is caused by nonlinearity of MZM modulator is investigated. In this case the EVM performance of multiband OFDM signals first reduced and then increased as the drive signal amplitude reached higher values. This experimental work supported the analysis that an optimum power level will improve the performance of wired-OFDM and MIMO-UFMC signals when they are subjected to MZM nonlinearity. Finally, the optical spectrum illustrated at optimum high and low power is provided in Fig. 11.

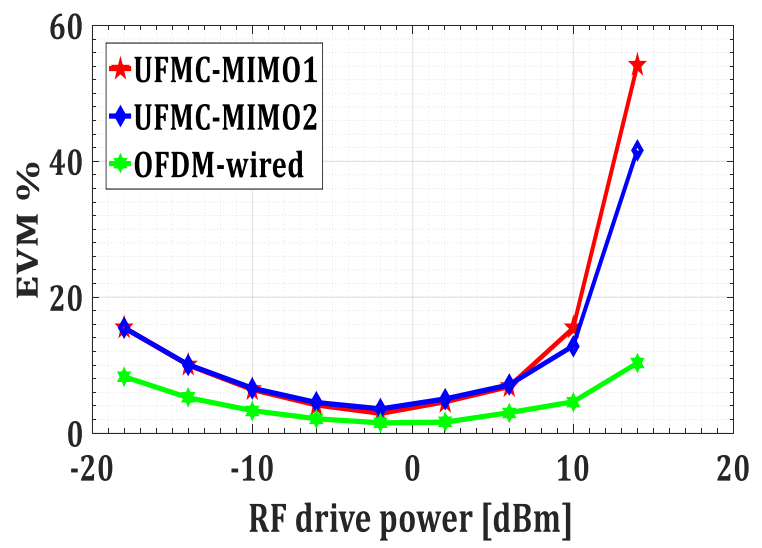

Fig. 10. RF drive power versus EVM.

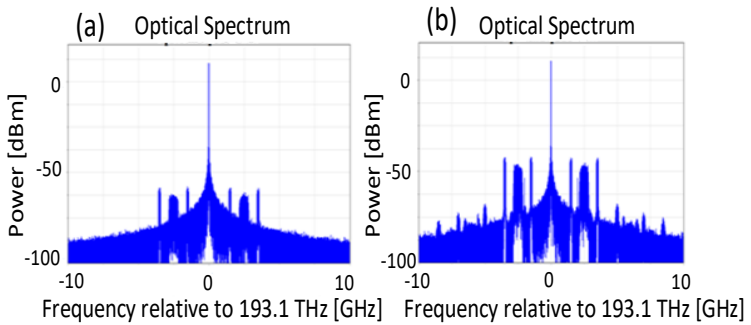

(c) Optical Spectrum

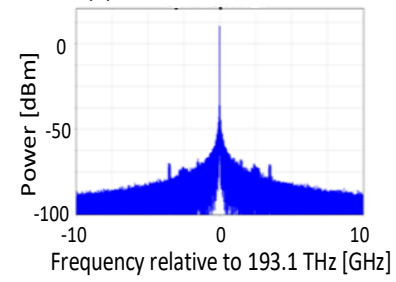

Fig. 11. Optical spectrum at (a) optimum power $-2 \mathrm{dBm}$ (b) high $\mathrm{RF}$ power $14 \mathrm{dBm}$ (c) low RF power $-14 \mathrm{dBm}$.

A theoretical analysis is presented in the Appendix to confirm the overall shape and frequency content obtained in the simulated optical spectra shown in Fig. 11 that is created due to the non-linearity of the MZM optical modulator shown in Fig. 4 at point b. It can be noted from consideration of equation (26) in the Appendix that the third and fifthorder non-linear distortion terms become apparent with high drive power. However, under the small signal condition third-order intermodulation is more dominant [48] as it was proven for multiband OFDM-ROF system in [47]. Therefore, in the analysis provided in the Appendix with small signal condition as in [47], the third-order intermodulations have a large probability to induce overlapping between wired-OFDM and MIMO-UFMC subcarriers leading to interband intermodulation distortion between the subcarriers in both signals bands causing interference from both signals on each other. For small drive power $\beta$, the non-linear distortion terms are negligible. However, the power of the optical carrier is much higher than the power of the optical carrier in OFDM and UFMC signals leading to poor system performance due to low received SNR. This situation may be understood from equation (28) in the Appendix where SNR depends on the signal power and also with low SNR the EVM performance will be high as displayed in equation (27). On the other hand, increasing the drive power increases the SNR resulting in improved system performance. Nevertheless, for very high drive power, the interband intermodulation terms in equation (26) will become prominent indicating increased interference between the UFMC and OFDM signals which will cause system performance degradation. From equation (28) it can be noted that as the distorted signal -which is caused by the thirdorder intermodulation- increases the SNR performance will decrease and then the EVM will be increased. Therefore, an optimum value of drive power will be required to provide the trade-off performance between the optical modulator nonlinear interband intermodulations and the SNR performance. Hence the theoretical development in the Appendix provides confidence in the results obtained from the simulation in relation to drive power optimization to obtain improved system performance.

\section{B. Downlink Fiber Transmission Performance}

The downlink network architecture, where both the wired-OFDM signal and the two $60 \mathrm{GHz}$ MIMO-UFMC are transmitted and received simultaneously through OFDM-PON system with 1:2 splitting ratio can be seen by referring back to Fig. 4. Data from the simulation provides the EVMverses received optical power characteristic for the two $60 \mathrm{GHz}$ 
MIMO-UFMC with 1 Gbps for each with B-T-B connection and after 20 $\mathrm{km}$ SSMF transmission. The received optical power is measured after the fiber transmission and before the photodetector. It can be noted from Fig. 12 that at the $5 \%$ EVM threshold the received optical powers at B-T-B for MIMO1and MIMO2 are $-3.4 \mathrm{dBm}$ and $-3.6 \mathrm{dBm}$, respectively. Hence there is $0.2 \mathrm{~dB}$ power difference between MIMO1 and MIMO2 which is caused by the finite input-output isolation of the doubled-balanced microwave mixers of the single sideband frequency translation process that result in a residual power level for MIMO1 at the original carrier frequency of $2.5 \mathrm{GHz}$ [25]. After transmission the receiver sensitivities for MIMO1 and MIMO2 are almost identical to their post-transmission values and there is almost no transmission penalty due to chromatic dispersion.

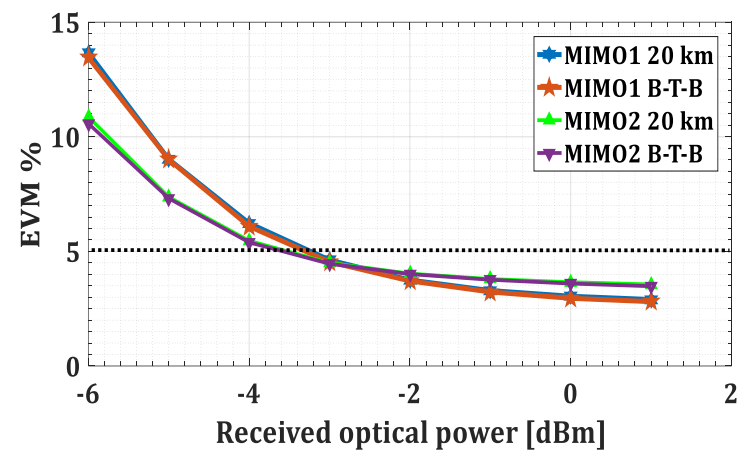

Fig. 12. EVM versus received optical power for the two $60 \mathrm{GHz}$ MIMOUFMC.

The bit error rate (BER) versus received optical power for wiredOFDM signal is shown in Fig. 13 where at BER $=10^{-9}$ the received power is $-14.2 \mathrm{dBm}$ with back-to-back transmission, Nevertheless, after $20 \mathrm{~km}$ SSMF transmission, the received power is almost the same as the $\mathrm{B}-\mathrm{T}-\mathrm{B}$ value. This result means that there is no penalty due to the chromatic dispersion. Therefore the performance obtained compares very well with more sophisticated and expensive architectures $[15,23$, 24].

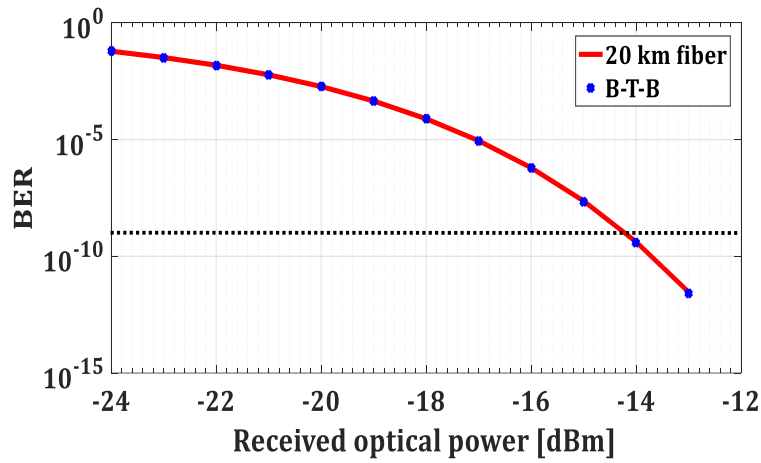

Fig. 13. BER versus received optical power for the wired-OFDM signal.

\section{Interference of Wireless Signals with Wired Signal}

It is important to investigate the interference due to intermixing between wired-OFDM and the two $60 \mathrm{GHz}$ RoF MIMO-UFMC signals in the new architecture, as future generations of mobile systems may utilize the UFMC waveform which is based on multicarrier modulation similar to OFDM $[49,50]$. In the co-existence scenario between wiredOFDM and RoF based on UFMC over PON there is, however, an effect that occurs as a consequence of the nonlinearity of the optical modulation known as interband intermodulation (IM). IM which results from different multicarrier signals induces both in-band interference and out-of-band emission between the multi-carrier signals leading to the degradation of the system performance [47]. In order to verify the successful transmission of the two $60 \mathrm{GHz}$ RoF MIMO-UFMC on the new network architecture only a minimal penalty should be obtained caused by the intermodulation intermixing with wired-OFDM signal. This situation is investigated from the simulation characteristic of EVM versus different received optical power levels which are shown in Fig. 14. At the $5 \%$ EVM threshold, the receiver sensitivities for nonconverged MIMO1 and MIMO2 are $-6.8 \mathrm{dBm}$ and $-7 \mathrm{dBm}$, respectively. However, converged MIM01 and MIMO2 sensitivities are reduced to $-6.2 \mathrm{dBm}$ and $-6.4 \mathrm{dBm}$, respectively. It can therefore be noted that a penalty of $0.6 \mathrm{~dB}$ occurs between the converged and non-converged system for both MIMO1 and MIMO2. This penalty is due to the IM distortion [47]. It can be seen, however, that the penalty is insignificant which indicated a very satisfactory performance for the new integrated network architecture.

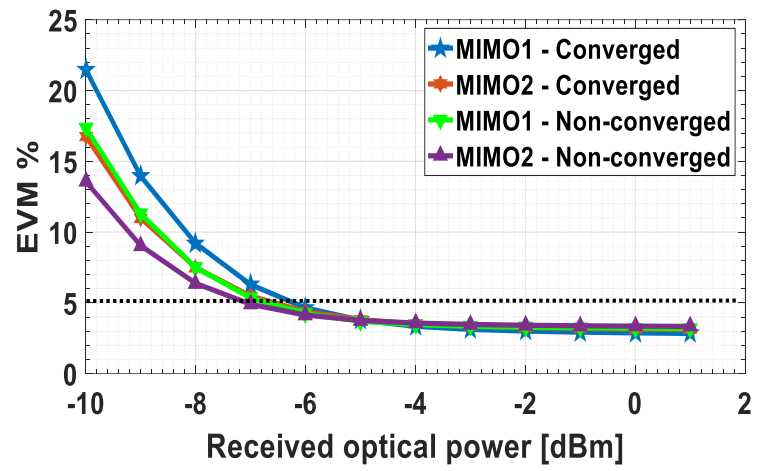

Fig. 14. EVM versus received optical power for the two $60 \mathrm{GHz}$ RoF MIMO-UFMC for converged and non-converged system.

\section{Conclusions}

A novel network architecture including two 5G MIMO-RoF signals based on UFMC and wired-OFDM over a standard PON has been described to provide simultaneous wired and wireless services for next generation access network applications. This architecture can be applied when using an OFDM-PON where efficient convergence of wired and wireless networks can be achieved. In addition, a single sideband frequency translation (SSB-FT) technique was utilized to produce a high spectrally efficient approach involving two MIMO-RoF and wired signals that are transmitted at the same carrier frequency. In particular, the approach produces a significant reduction in the complexity of the transceiver design where only one LO is required enabling simultaneous transmission of two RoF-MIMO and wired signals over a standard OFDM-PON. Furthermore, a receiver carrier self-heterodyne technique was adapted in order to reuse the remote optical LO as a LO to downconvert the $60 \mathrm{GHz}$ MMW at the ONU which also produces an additional cost reduction for the network.

Network simulation performance characteristics demonstrate that the two $60 \mathrm{GHz}$ MIMO-UFMC signals with 16 QAM and $1 \mathrm{Gbps}$ for each MIMO provide good performance after transmission over $20 \mathrm{~km}$ of SSMF and splitting ratio 1:2. In addition, a wired-OFDM signal with 16 $\mathrm{QAM}$ and 2.5 Gbps provides very good performance at a $\mathrm{BER}=10^{-9}$, where negligible penalty is obtained due to chromatic dispersion over the $20 \mathrm{~km}$ SSMF. Furthermore, the intermixing effect observed from the simulation characteristics gave a $0.6 \mathrm{~dB}$ penalty between converged and non-converged networks with an EVM equal to $5 \%$. Hence this aspect from the simulation results also supports the proposition that the new 
architecture is a promising candidate for next generation converged wired and wireless access networks. It should be noted, however, that a useful area for future work will be the comparison of remote with local optical heterodyning in relation to wireless channel impairment.

Finally, the convergence approach which utilizes the SSB-FT technique enables simultaneous transmission of wired and MIMO-RoF signals over the OFDM-PON infrastructure. As efficient convergence with high spectral efficiency and low cost deployment was therefore demonstrated with this novel network architecture, it could also be expanded to support multiple MIMO systems to meet the future 5G network requirements [51].

\section{Appendix}

A theoretical analysis focused on the non-linearity of the MZM optical modulator shown in Fig. 4 at point (b) that has an effect on the simulated optical spectrum shown in Fig. 11 is presented here. The non-linear transfer function of the single drive MZM is written as [52]:

$$
\frac{P_{o u t}(t)}{P_{\text {in }}(t)}=\frac{1}{2}\left[1+\cos \left(\frac{\pi V_{\text {in }}(t)}{V_{\pi}}\right)\right]
$$

where $P_{\text {in }}(t)$ and $P_{\text {out }}(t)$ represent the power of the input carrier and the power of the single drive MZM respectively. $V_{\pi}$ is the half-wave switching voltage of the MZM. $V_{i n}(t)$ is a function of the bias voltage and the input signal.

In the new network architecture, the input signal is the aggregation of the two MIMO-UFMC and OFDM signals as expressed in the following:

$$
V_{\text {in }}(t)=V_{\text {bias }}+S_{\text {aggregated }}(t)
$$

Since the MZM operates in quadrature, the bias point in the network $V_{\text {bias }}$ will be equal to:

$$
V_{\text {bias }}=\frac{V_{\pi}}{2}
$$

$S_{\text {aggregated }}(t)$ is the accumulated RF derived signals into the MZM comprising two MIMO UFMC signals and the OFDM wired-PON signal expressed as:

$$
S_{\text {aggregated }}(t)=\left[S_{\text {MIMO2 }}(t)+S_{\text {wired }-O F D M}(t)+S_{\text {MIMO1 }}(t)\right]
$$

In addition, the baseband wired-OFDM signal can be written as [53]:

$$
S_{\text {wired-OFDM }}(t)=\sum_{k=0}^{K-1} c_{k} e^{j 2 \pi f_{k} t}
$$

where $c_{k}$ represents the $k_{t h}$ subcarrier and $f_{k}$ is the frequency of $k_{t h}$ subcarrier.

In the simulation the baseband OFDM signal is upconverted to a radio frequency $f_{R F}$ equal to $2.5 \mathrm{GHz}$ and thus the OFDM signal can be expressed as:

$$
S_{U p-\text { wired-OFDM }}(t)=S_{\text {wired-OFDM }}(t)\left(A e^{j 2 \pi f_{R F} t}\right)
$$

where $A$ and $f_{R F}$ are the amplitude and radio frequency for the upconverted OFDM signal.

The UFMC baseband signal according to the UFMC transceiver illustrated in Fig. 1 is [54]:

$$
S_{U F M C}(t)=\sum_{i=0}^{B} \sum_{l=0}^{L-1} \sum_{k=0}^{K-1} c_{k}^{i} e^{j 2 \pi f_{k}(t-l)} F_{i}(l)
$$

where $B$ is denoted as the subbands of the available UFMC bandwidths. $L$ is FIR filtering length which is applied to each subband modulated signal and $K$ represents the group of subcarriers. The upconverted UFMC signal, which represents MIM01 and MIMO2, can be written as:

$$
S_{U p-U F M C}(t)=S_{U F M C}(t)\left(A e^{j 2 \pi f_{R F} t}\right)
$$

By applying the single sideband frequency translation technique, which is shown in Fig. 4, the output of each mixer will be equivalent to:

$$
S_{U F M C}(t)\left[\left(A e^{j 2 \pi\left(f_{R F}+f_{L O}\right) t}\right)+\left(A e^{j 2 \pi\left(f_{R F}-f_{L O}\right) t}\right)\right]
$$

Two bandpass filters are used to select the upper and lower sidebands which represent MIMO1 and MIMO2 using center frequencies equal to $f_{R F}+f_{L O}$ and $f_{R F}-f_{L O}$ respectively where $f_{R F}=2.5 \mathrm{GHz}$ and the local oscillator frequency $f_{L O}=1 \mathrm{GHz}$. Therefore, the two MIMO signals can be expressed by:

$$
\begin{aligned}
& S_{\text {MIMO1 }}(t)=S_{U F M C}(t)\left(A e^{j 7 \pi \times 10^{9} t}\right) \\
& S_{M I M O 2}(t)=S_{U F M C}(t)\left(A e^{j 3 \pi \times 10^{9} t}\right)
\end{aligned}
$$

After the substitution of equations (13), (17) and (18) in equation (11) it becomes:

$$
S_{\text {aggregated }}(t)=\left[\begin{array}{l}
S_{U F M C}(t)\left(A e^{j 3 \pi \times 10^{9} t}\right) \\
+S_{\text {wired-OFDM }}(t)\left(A e^{j 5 \pi \times 10^{9} t}\right) \\
+S_{U F M C}(t)\left(A e^{j 7 \pi \times 10^{9} t}\right)
\end{array}\right]
$$

Equation (19) can then be written as:

$$
S_{\text {aggregated }}(t)=A S_{T}(t)
$$

Substituting equations (10) and (20) in equation (8) it becomes:

$$
\frac{P_{\text {out }}(t)}{P_{\text {in }}(t)}=\frac{1}{2}\left[1+\cos \left(\frac{\pi}{V_{\pi}} V_{\text {bias }}+\frac{\pi}{V_{\pi}} A S_{T}(t)\right)\right]
$$

By assuming $\alpha=\frac{\pi}{V_{\pi}} V_{\text {bias }}$ and $\beta=\frac{A}{V_{\pi}}$ where $\alpha$ and $\beta$ are the normalized bias and drive levels, respectively since the drive power approximately equals to $P \approx A^{2}$ so $\beta=\frac{\sqrt{P}}{V_{\pi}}$. Therefore substituting $\alpha$ and $\beta$ values in equation (21) yields:

$$
\frac{P_{\text {out }}(t)}{P_{\text {in }}(t)}=\frac{1}{2}\left[1+\cos \left(\alpha+\beta \pi S_{T}(t)\right)\right]
$$

The cosine is expanded to obtain the following:

$$
\frac{P_{o u t}(t)}{P_{\text {in }}(t)}=\frac{1}{2}\left[\begin{array}{l}
1+\cos (\alpha) \cos \left(\beta \pi S_{T}(t)\right) \\
-\sin (\alpha) \sin \left(\beta \pi S_{T}(t)\right)
\end{array}\right]
$$

After substituting the expanded values for $\alpha$ and $\beta$ in equation (23) it then yields: 


$$
\frac{P_{\text {out }}(t)}{P_{\text {in }}(t)}=\frac{1}{2}\left[1-\sin \left(\beta \pi S_{T}(t)\right)\right]
$$

Then expanding equation (24) into a Taylor series gives:

$$
\frac{P_{o u t}(t)}{P_{\text {in }}(t)}=\frac{1}{2}\left[1-\sum_{n=0}^{\infty} \frac{(-1)^{n}}{(2 n+1) !}\left(\beta \pi S_{T}(t)^{2 n+1}\right)\right]
$$

By neglecting the higher-order terms, the MZM power transfer function can finally be approximated as:

$$
\frac{P_{\text {out }}(t)}{P_{\text {in }}(t)} \approx \frac{1}{2}\left[1-\beta \pi S_{T}(t)+\frac{1}{3 !} \beta \pi S_{T}(t)^{3}-\frac{1}{5 !} \beta \pi S_{T}(t)^{5}\right]
$$

Equation (26) can be related to the performance matrices that have been used in the simulation with particular reference to the EVM characteristics. As the SNR is largely dependent on the intermodulation products caused by nonlinearity of the MZM and therefore the EVM relies on the SNR system performance. Based on [55] EVM can be expressed as:

$$
E V M \approx \frac{1}{\sqrt{S N R}}
$$

where SNR represents the power of the received signal $P_{r x}$ divided by the total signal noise. In this analysis the total signal noise can be assumed as the additive of receiver noise power $P_{n}$ and the power of the distorted signal $P_{\text {distortion }}$ which is the power of the distorted signal that is caused by the nonlinearity of the MZM modulator and it can be presented by the third and fifth order intermodulation distortion as shown in equation (26). Hence the SNR can be written as:

$$
S N R=\frac{P_{r x}}{P_{n}+P_{\text {distortion }}}
$$

\section{Funding Information}

This work was funded by TM R\&D Research Grant under Grant No. RDTC/160914.

\section{Acknowledgement}

The authors thank Dr. Redhwan Shaddad from Taiz University -Yemen for his assistance.

\section{References}

1. S. Alavi, M. Soltanian, I. Amiri, M. Khalily, A. Supa'at, and H. Ahmad, "Towards 5G: A photonic based millimeter wave signal generation for applying in 5G access fronthaul," Scientific reports, vol. 6, 2016.

2. C. Sacchi, T. F. Rahman, I. A. Hemadeh, and M. El-Hajjar, "MillimeterWave Transmission for Small-Cell Backhaul in Dense Urban Environment: A Solution Based on MIMO-OFDM and Space-Time Shift Keying (STSK)," IEEE Access, vol. 5, pp. 4000-4017, 2017.

3. U. Habib, A. E. Aighobahi, C. Wang, and N. J. Gomes, "Radio over fiber transport of mm-Wave $2 \times 2$ MIMO for spatial diversity and multiplexing," in IEEE International Topical Meeting on Microwave Photonics (MWP), pp. 39-42, 2016.

4. G. Carpintero, R. C. Guzmán, C. Gordón, G. Kervella, M. Chitoui, and F. Van Dijk, "Photonic integrated circuits for radio-frequency signal generation," Journal of Lightwave Technology, vol. 34, pp. 508-515, 2016.
5. T. Dahawi, Z. Yusoff, M. Salleh, and J. Senior, "OFDM Transmission in A Converged $60 \mathrm{GHz}$ MMW RoF over OFDM-PON," in $7^{\text {th }}$ IEEE International Conference on Photonics (ICP), pp. 1-3, 2018.

6. A. Hammoodi, L. Audah, and M. A. Taher, "Green coexistence for $5 G$ waveform candidates: a review," IEEE Access, vol. 7, pp. 10103-10126, 2019.

7. Y. Cai, Z. Qin, F. Cui, G. Y. Li, and J. A. McCann, "Modulation and multiple access for $5 \mathrm{G}$ networks," IEEE Communications Surveys \& Tutorials, vol. 20, pp. 629-646, 2018.

8. M. Mukherjee, L. Shu, V. Kumar, P. Kumar, and R. Matam, "Reduced outof-band radiation-based filter optimization for UFMC systems in 5G," in International Conference on Wireless Communications and Mobile Computing (IWCMC), pp. 1150-1155, 2015.

9. P. Del Fiorentino, C. Vitiello, V. Lottici, F. Giannetti, and M. Luise, "A robust resource allocation algorithm for packet BIC-UFMC 5G wireless communications," in $24^{\text {th }}$ European signal processing conference (EUSIPCO), pp. 843-847, 2016.

10. G. Kongara, L. Yang, C. He, and J. Armstrong, "A Comparison of CPOFDM, PCC-OFDM and UFMC for $5 \mathrm{G}$ Uplink Communications," arXiv preprint arXiv:1810.04840, 2018.

11. W. Yongxue, W. Sunan, and W. Weiqiang, "Performance Analysis of the Universal Filtered Multi-Carrier (UFMC) Waveform for 5 G System," in Journal of Physics: Conference Series, p. 012065, 2019.

12. R. S. Yarrabothu and U. R. Nelakuditi, "Optimization of out-of-band emission using kaiser-bessel filter for UFMC in $5 \mathrm{G}$ cellular communications," China Communications, vol. 16, pp. 15-23, 2019.

13. S. Buzzi, C. D'Andrea, D. Li, and S. Feng, "MIMO-UFMC transceiver schemes for millimeter-wave wireless communications," IEEE Transactions on Communications, vol. 67, pp. 3323-3336, 2019.

14. A. Delmade, C. Browning, A. Farhang, N. Marchetti, L. E. Doyle, R. D. Koilpillai, et al., "Performance analysis of analog IF over fiber fronthaul link with 4G and 5G coexistence," Journal of Optical Communications and Networking, vol. 10, pp. 174-182, 2018.

15. H. N. Parajuli, E. Udvary, and J. Poette, "Experimental Demonstration of the Potential 5G Based Multiplexed Radio Frequency Signals Transmission in Passive Optical Network," Radioengineering, vol. 29, p. 439, 2020.

16. A. T. Latunde, A. Papazafeiropoulos, P. Kourtessis, and J. M. Senior, "Coexistence of OFDM and FBMC for resilient photonic millimeter-wave 5G mobile fronthaul," Photonic Network Communications, vol. 37, pp. 335348, 2019.

17. H. Uzawa, K. Honda, H. Nakamura, and J. Terada, "Optical access technologies for mobile fronthaul in $5 \mathrm{G}$ and beyond," in Broadband Access Communication Technologies XIV, p. 1130702, 2020.

18. X. Liu, "Optical Transport and Access Technologies for $5 \mathrm{G}$ Wireless," in Asia Communications and Photonics Conference, p. M3C. 1, 2019.

19. B. Hadjer and B. Abdelhafid, "Comparison \& performance evaluation of MIMO-FBMC and MIMO-UFMC systems for various equalization techniques," in International Conference on Networking and Advanced Systems (ICNAS), pp. 1-5, 2019.

20. Y. Xiang, C. Chen, C. Zhang, and K. Qiu, "Wired/wireless access integrated RoF-PON with scalable generation of multi-frequency MMWs enabled by polarization multiplexed FWM in SOA," Optics express, vol. 21, pp. 1218-1225, 2013.

21. C. Chow, C. Yeh, J. Sung, and C. Hsu, "Wired and wireless convergent extended-reach optical access network using direct-detection of alloptical OFDM super-channel signal," Optics Express, vol. 22, pp. 3071930724, 2014.

22. M. Elmagzoub, A. B. Mohammad, R. Q. Shaddad, and S. A. Al-Gailani, "New RoF-PON architecture using polarization multiplexed wireless MIMO signals for NG-PON," Optics Communications, vol. 344, pp. 55-64, 2015.

23. M. Elmagzoub, A. B. Mohammad, R. Q. Shaddad, and S. A. Al-Gailani, "Polarization multiplexing of two MIMO RoF signals and one baseband 
signal over a single wavelength," Optics \& Laser Technology, vol. 76, pp. 70-78, 2016

24. R. Zhang and J. Ma, "Full-duplex link with a unified optical OFDM signal for wired and millimeter-wave wireless accesses based on direct detection," Optical Switching and Networking, vol. 25, pp. 33-39, 2017.

25. C.-P. Liu and A. J. Seeds, "Transmission of wireless MIMO-type signals over a single optical fiber without WDM," IEEE Transactions on Microwave Theory and Techniques, vol. 58, pp. 3094-3102, 2010.

26. M. M. Abayomi T. Latunde, Pandelis Kourtessis, and John Senior "OQAM-OFDM RoF with IM-DD Remote Heterodyne $28 \mathrm{GHz}$ Upconversion for $5 \mathrm{G}$ Millimeter RANs " presented at the International Conference on Transparent Optical Networks (ICTON) Trento, Italy 2016.

27. C. Browning, A. Farhang, A. Saljoghei, N. Marchetti, V. Vujicic, L. Doyle, et al., "5G Wireless and Wired Convergence in a Passive Optical Network using UF-OFDM and GFDM," arXiv preprint arXiv:1703.01956, 2017.

28. J. Pérez Santacruz, S. Rommel, U. Johannsen, A. Jurado-Navas, and I. Tafur Monroy, "Candidate Waveforms for ARoF in Beyond 5G," Applied Sciences, vol. 10, p. 3891, 2020.

29. G. Wunder, P. Jung, M. Kasparick, T. Wild, F. Schaich, Y. Chen, et al., "5GNOW: non-orthogonal, asynchronous waveforms for future mobile applications," IEEE Communications Magazine, vol. 52, pp. 97-105, 2014.

30. F. Schaich, B. Sayrac, S. Elayoubi, I. P. Belikaidis, M. Caretti, A. Georgakopoulos, et al., "FANTASTIC-5G: flexible air interface for scalable service delivery within wireless communication networks of the $5^{\text {th }}$ generation," Transactions on Emerging Telecommunications Technologies, vol. 27, pp. 1216-1224, 2016.

31. M. H. Habaebi, J. Chebil, A. Al-Sakkaf, and T. Dahawi, "Comparison between scheduling techniques in long term evolution," IIUM Engineering Journal, vol. 14, 2013

32. J. Wen, J. Hua, W. Lu, Y. Zhang, and D. Wang, "Design of waveform shaping filter in the UFMC system," IEEE Access, vol. 6, pp. 32300-32309, 2018.

33. M.-F. Tang and B. Su, "Filter optimization of low out-of-subband emission for universal-filtered multicarrier systems," in IEEE International Conference on Communications Workshops (ICC), pp. 468473, 2016

34. W. Rong, J. Cai, and X. Yu, "Low-complexity PTS PAPR reduction scheme for UFMC systems," Cluster Computing, vol. 20, pp. 3427-3440, 2017.

35. M. Morant, J. Prat, and R. Llorente, "Radio-over-fiber optical polarization-multiplexed networks for 3GPP wireless carrier-aggregated MIMO provision," Journal of Lightwave Technology, vol. 32, pp. 37213727, 2014.

36. I. Seto, H. Shoki, and S. Ohshima, "Optical subcarrier multiplexing transmission for base station with adaptive array antenna," IEEE Transactions on Microwave Theory and Techniques, vol. 49, pp. 20362041, 2001.

37. J. Capmany, B. Ortega, A. Martinez, D. Pastor, M. Popov, and P. Fonjallaz, "Multiwavelength single sideband modulation for WDM radio-over-fiber systems using a fiber grating array tandem device," IEEE photonics technology letters, vol. 17, pp. 471-473, 2005.

38. Y. Yang, M. J. Crisp, R. V. Penty, and I. H. White, "Low-cost MIMO radio over fiber system for multiservice DAS using double sideband frequency translation," Journal of Lightwave Technology, vol. 34, pp. 3818-3824, 2016.

39. P. T. Dat, A. Kanno, N. Yamamoto, and T. Kawanishi, "Simultaneous transmission of 4G LTE-A and wideband MMW OFDM signals over fiber links," in IEEE International Topical Meeting on Microwave Photonics (MWP), pp. 87-90, 2016

40. P. T. Dat, A. Kanno, K. Inagaki, and T. Kawanishi, "High-Capacity Wireless Backhaul Network Using Seamless Convergence of Radio-over-Fiber and 90-GHz Millimeter-Wave," Journal of Lightwave Technology, vol. 32, pp. 3910-3923, 2014/10/15 2014.

41. M. A. Ilgaz and B. Batagelj, "Using Tunable Dispersion-Compensated Modules to Overcome the Power Penalty of a Millimeter-Wave OptoElectronic Oscillator Signal that is Distributed via a Passive Optical
Network for 5G Networks," in $11^{\text {th }}$ International Symposium on Communication Systems, Networks \& Digital Signal Processing (CSNDSP), pp. 1-6, 2018.

42. C. Lim, Y. Tian, C. Ranaweera, A. Nirmalathas, E. Wong, and K.-L. Lee, "Evolution of Radio-over-Fiber Technology," Journal of Lightwave Technology, 2018.

43. R. Sardiñas-Fernández, A. García-Juárez, I. Zaldívar-Huerta, L. GonzálezMondrangón, L. Quintero-Rodríguez, and E. Avilez-Valenzuela, "Generation of microwave signals by heterodyning and multiplication techniques: a comparison," in Terahertz, RF, Millimeter, and Submillimeter-Wave Technology and Applications XII, p. 1091710, 2019.

44. T. Dahawi, Z. Yusoff, R. Shaddad, M. Salleh, and J. Senior, "Converged Radio Over Fiber and OFDM-PON based on Single-Sideband Frequency Translation Technique," International Journal of Technology, vol. 10, p. 1365, 11/27, 2019.

45. B. Lin, J. Li, H. Yang, Y. Wan, Y. He, and Z. Chen, "Comparison of DSB and SSB transmission for OFDM-PON," Journal of Optical Communications and Networking, vol. 4, pp. B94-B100, 2012.

46. X. Liu, "An Investigation about Optical Millimeter-wave Generation Technology," in Proceedings of the 2020 International Conference on Pattern Recognition and Intelligent Systems, pp. 1-6, 2020.

47. J. Wang, C. Liu, J. Zhang, M. Zhu, M. Xu, F. Lu, et al., "Nonlinear interband subcarrier intermodulations of multi-RAT OFDM wireless services in $5 \mathrm{G}$ heterogeneous mobile fronthaul networks," Journal of Lightwave Technology, vol. 34, pp. 4089-4103, 2016.

48. S. V. Zhidkov, "Performance analysis of multicarrier systems in the presence of smooth nonlinearity," EURASIP Journal on Wireless Communications and Networking, p. 281080, 2004.

49. T. 3GPP. Study on New Radio (NR) Access Technology Physical Layer Aspects: [Online]. Available: https: // portal. 3gpp.org/ desktopmodules / Specifications/SpecificationDetails.aspx, [Accessed: May-2017].

50. T. Inoue, "5G standards progress and challenges," in IEEE Radio and Wireless Symposium (RWS), pp. 1-4, 2017.

51. A. Ghosh, A. Maeder, M. Baker, and D. Chandramouli, " $5 \mathrm{G}$ evolution: A view on $5 \mathrm{G}$ cellular technology beyond $3 \mathrm{GPP}$ release 15," IEEE Access, vol. 7, pp. 127639-127651, 2019.

52. C.-T. Lin, J. J. Chen, S.-P. Dai, P.-C. Peng, and S. Chi, "Impact of nonlinear transfer function and imperfect splitting ratio of MZM on optical upconversion employing double sideband with carrier suppression modulation," Journal of Lightwave Technology, vol. 26, pp. 2449-2459, 2008.

53. J. Armstrong, "OFDM for optical communications," Journal of lightwave technology, vol. 27, pp. 189-204, 2009.

54. T. Wild and F. Schaich, "A reduced complexity transmitter for UFOFDM," in IEEE 81st Vehicular Technology Conference (VTC Spring), pp. 1-6, 2015.

55. S. Zhou, Y. Song, Y. Tan, L. Zhang, Y. Li, J. Ye, et al., "Performance of optical OFDM transmission over RoF system with Mach-Zehnder modulator," in 2011 Asia Communications and Photonics Conference and Exhibition (ACP), pp. 1-6, 2011 